\title{
ARTIFICIAL INTELLIGENCE-FACIAL RECOGNITION
}

\author{
Shashank Jagadeesha ${ }^{1}$

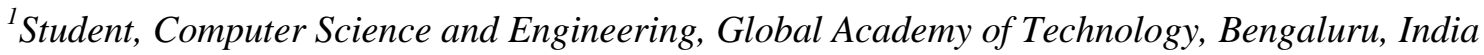

\begin{abstract}
The goal is to revealing more information about the self or stranger in the public through AI-Facial Recognition. By just holding your phone up to someone's face you can check out the backgrounds of strangers in the street and seeing links to their social networks. The application even helps in the facial recognition of the criminals or terrorists which helps the security forces in locating them, where the entire information of the stranger Face recognition data will be stored in the database or cloud using data distribution technique for security applications. This to generalise the high-end safety of the public even through your mobiles.
\end{abstract}

Keywords: Face detection ${ }^{1}$, Face recognitions $^{2}$, Information matching ${ }^{3}$, Database $^{4}$, Cloud computing $^{5}$, Privacy $^{6}$, Hadoop ${ }^{6}$.

\section{INTRODUCTION}

Facial recognition is a biometric method of identifying an individual by comparing live capture or digital image data with the stored record for that person. Facial recognition systems are commonly used for security purposes and is now rapidly increasing in other fields too .

Person authentication from face verification is considered to be a passive and one of the most nonintrusive modalities of biometrics. For biometric application we need to identify a particular person in real time whose face image is already registered in the system. For proper verification, the probe image should exactly match to the registered image of that particular person.

The Hadoop schema is formed on viably procedure data-intensive MapReduce requisitions. Hadoop clients point out the provision calculation rationale as far as a map, what's more on decrease function, which would often termed MapReduceprovisions[2].

The idea of data-locality has also its effect on the execution of Hadoop would way factors, since they influence the execution in the guide stage when planning assignments. The assignmentplanning techniques for Hadoopought further bolstering seemingly. Think as of information area should upgrade. However, it gives more weight to the pixels which are often a part of the edge and counts pixels that are unmatched between query and database images. This measure has been tested on a publicly available database and also on a database that was developed at IIT Kanpur. The Results throughseries of experiments show that the proposed idea gave a high recognition rate of nearly $100 \%$ for the first likely matched faces on databases [7] which are publicly available .

\section{EXISTING SYSTEMS}

\subsection{Face Recognition Application Fields?}

The following are some example of facebased surveillance. To enhance town centre surveillance in Newham Borough of London, this has 300 cameras linked to the closed circuit TV (CCTV) controller room. The city council claims that the technology has helped to achieve a little less of 50\%drop in crime because of its use.Samekind of systems is in Birmingham, England. In 1999 Visionics was awarded a contract from National Institute of Justice to develop smart CCTV technology.Applications[1]

\begin{tabular}{|l|l|}
\hline Fields on use & Implementation \\
\hline Social-Medias & $\begin{array}{l}\text { Easy of connecting to people } \\
\text { (on facebook, instagram) } \\
\text { Identifying Tv shows } \\
\text { Profile searching(facebook) }\end{array}$ \\
\hline Information security & $\begin{array}{l}\text { Permission based systems } \\
\text { Criminals database(OS, data } \\
\text { bases) } \\
\text { User authentication(on line } \\
\text { banking) }\end{array}$ \\
\hline Biometrics & $\begin{array}{l}\text { Person identifica- } \\
\text { tion(,Aadhar card, pass- } \\
\text { ports,voter ID,DL) } \\
\text { Automatic identity verifica- } \\
\text { tion(in border force ) }\end{array}$ \\
\hline Personal security & $\begin{array}{l}\text { Home video surveillance } \\
\text { systems identification } \\
\text { Expression } \\
\text { (driver monitoring system) } \\
\text { Posture of the person }\end{array}$ \\
\hline Entertainment & $\begin{array}{l}\text { video game systems } \\
\text { Photo camera applications }\end{array}$ \\
\hline
\end{tabular}




\section{a). Social-Medias}

Facebook's automatic tag suggestion feature and profile searching, which uses face recognition to suggest people you might want to tag in your photos or the people whom they wanted to stalk have become easier in these year. Face recognition for people tagging certainly saves time. It is can seen now in the softwares like Apple's iPhoto, Picasa and on Facebook.

Further one of the apps called 'Shazam for TV', TVtak is an application that identifies the TV show you're watching, simply by pointing your iPhone's camera near the screen. Within no time, it will work out exactly the show or advertise that you are watching.

\section{b). Information Security}

Face recognition could one day replace the physical patrolling or chasing in search of terrorists by the security forces through our mobiles - imagine you take a photo of the stranger on whom you have doubt, just by logging into the software with the image, you will get the entire information of the stranger. Face recognition technology for security applicationswill so advanced such that ,It's not fooled by photographs. Well-known companies are reportedly interested in integrating the technique into their products also.

The law enforcement agencies were the first companies to invest on such researches - e.g. the Woodrow W.Bledsoecase was the first case to be examined.

\section{c). Biometrics}

Security cameras are presently common in airports, apartments, multinational companies,colleges, ATM, Bank and in any locations with a security system. Face recognition is a biometricsystem used to identify or verify a person from a digital image. Face Recognitionsystem is used in even in the border security for more confidential purposes. Even the Person identification like Adhaar card,passports,election Id cards,DL are also equipped with AI-face recognition

\section{d). Personal Security}

Where the persons mood, his health graph will be checked by his facial expressions.

Many Companies, have been using such applications for more safety purposes, such as Toyota are inventing sleep detectors in the cars to increase safety of the driver.[4]

\section{e). Entertainment}

Nowadays different enterprises are using face recognition in their products. One good example is automated face recognition in entertainment business. Products like Project Natal(by Microsoft) or PlayStation games(by Sony)[5] uses Eye for face recognition. And alsoMicrosoft'sKinect's[6] propelled movement sensing abilities need provided for those Xbox 360 an entire new terms of the lease for life What's more opened up gaming will new gatherings of people
Eventually Tom's perusing totally getting rid of equipment controllers.

\section{How it Works?}

The following four-stage process illustrates the way in which the biometric system operates.

Capture-a physical behaviour or behavioural samples is captured by the system durigenrollment.

Extraction - unique data is extracted from the sample and a template is created.

Comparision -the template is the then compared with a new sample.

Matching - the system then manages to compare image from the extracted information to check matchability.

When the user faces the cameras, standing about a few distance from the camera. The system will locate the users face and perform the matching with respect to the doubted identity in the secured database.

In order to develop a face recognition system several factors need to be taken in hand.

1. The overall speed of the system from detection to recognition should be acceptable.

2. The accuracy should be high.

3. The system should be easily updated and enlarged, that is easy to increase.

However the simple general face recognition system can be described as follows. The input of a face recognition system is always an image or video stream. The output is an identification or verification of the subject or subjects that appear in the image or video. The Face Detection and Feature Extraction phases could run simultaneously. We define face recognition as a 3-steps as see Fig-1.

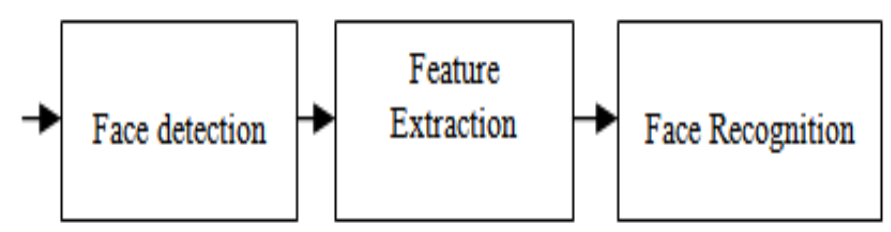

Fig-1: A general face recognition system.

Face detection is defined as the process of extracting faces from scenes. So, the system positively identifies a certain image region as a face. The next step -feature extractioninvolves obtaining relevant facial features from the data. These features could be certain face regions, variations, angles or measures, which can be human relevant emotion recognition or not. Finally, the system does recognize the face. In an identification task, the system would report an identity from a database[3].

\subsection{Algorithm}

Mainly there are two types of Face Recognition algorithm, 1.Image-Based Face Recognition Algorithm and

2.Video-Based Face Recognition Algorithms. 
Here we shall discuss about Image-Based Face Recognition Algorithms.

There are numerous Image-Based Face recognition algorithms using which the working process of the face recognition system can be carried out in different ways.

some of them are PCA, ICA, LDA, EP, EBGM, Kernel Methods , Trace Transform AAM , 3-D Morphable Model , 3-D Face Recognition, Bayesian Framework, SVM, HMM Boosting \& Ensemble, Algorithms Comparisons.

\section{PCA}

Determined starting with Karhunen-Loeve'sconversion. Provided for a s-dimensional vector representational about each face to An preparation set for images, vital part Investigation (PCA) has a tendency will find An t-dimensional subspace whose support vectors relate of the greatest difference bearing in the first picture space. This new subspace will be regularly easier dimensional $(\mathrm{t}<<\mathrm{s})$. Whether those picture elements would viewed as irregular variables, those PCA groundwork vectors are characterized Concerning illustration eigenvectors of the scramble grid.

\section{ICA}

Independent Component Analysis (ICA) minimizes both second-order and higher-order dependencies in the data information What's more endeavors to discover those support along which the information (when anticipated onto them) need aid - statistically free. Bartlett et al. Gave two architectures from claiming ICA to face distinguishment task:

\section{Building design}

i - statistically free groundwork images, and. Construction modelling

ii - factorial code representational.

so on...

\section{PROPOSED IDEA}

What if mobile uses hi-def cameras to get connected with new people and to get information about strangers?

There are numerous social medias like facebook, instagram, etc. will now recognises overwhelming number of faces from camera-equipped Artificial Intelligence.

Facial recognitions are used normally in mobiles for unlocking purposes, it can also be used for scanning strangers faces too, for having information about his EMOTION, ETHNICITY, AGE, LOCATION AND EXISTENCE OF ANY OF THE SOCIAL ACCOUN .The Fig-2 show the following information.

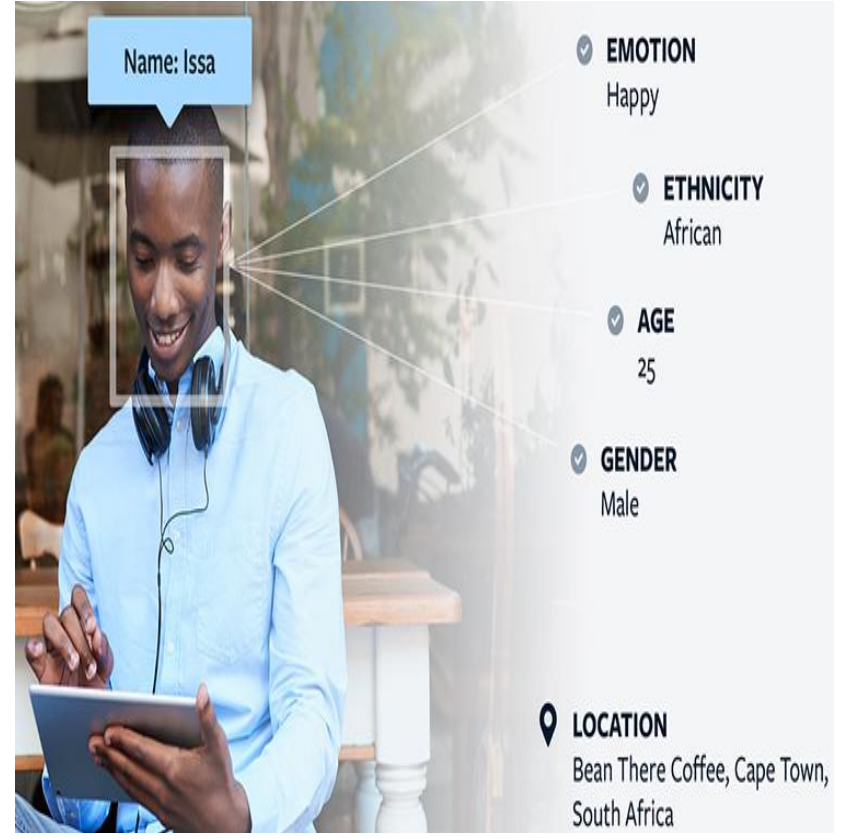

Fig-2: Information sowed after face detection

According to the statistics there are 2.1 billion monthly active users of face book and 1.6 billion uploads of photos daily and similarly instagram statistics shows that there are 375 million monthly active users and 161 million stories uploaded daily. Having this vast information it becomes easy to connect with these social medias easily.

It's certainly a complicated one. Like when your mobile gets into wrong hands or recognises new person and other possibilities which undergoes new facial recognition. For instance, think of bus stop scenario, when a person asks for details about the arrival and leaving of buses, after with a continuous conversation you certainly don't want to become friends without knowing strangers background. Now we can have the help of AI facial -recognition, by just his facial recognition you will get to know about that person ,if he is in any of the social media account. If yes, you can get the information present in the personal bio which a person has filled in while creating that account.

This would be authentic only if the person face recognition is matched with facial record present in the social media account.

\section{Advantage}

If $\mathrm{u}$ want to know whether the person is delectable, legitimate in-order to make friendship with the stranger by gathering personal information present on his social media account. If that person feels agreeable to you without typing his name through the AI face recognition you can connect with that persons social media account by just a click from the pop-up message you get once the recognition is finished and if the persons account is present on that social website.

It basically gives hint to you whether the person is present on that social media or not. 
Any time your facial data may be stored not only by the facial record in the facebook and instagram, there's a possibility even getting the information through your ration cards, adhaar cards, national membership card, passport ,etc.which will be linked even to it.

Love it or hate it this technology is going to have greater advantage.

In addition to it, a significant combination alerting the patrol can be added to the application but the product may seem like an odd choice, but the result would have greater advantage.

What if all digital place signage used hi-def cameras to target criminals in the street?

You're at public places, for example at bus stop the TVs and LED displays have the stored cameras. This data is cross-referenced with other databases that contains facial data information of the person. The face of person is now becomes the epicentre for retargeting unit as shown in the Fig-3. For instance, even your camera equipped AI-face recognition can also plays a vital role if it has captured the facial record of the criminal person it will be verified with the different database units all over the world, which has filed records of the terrorists.

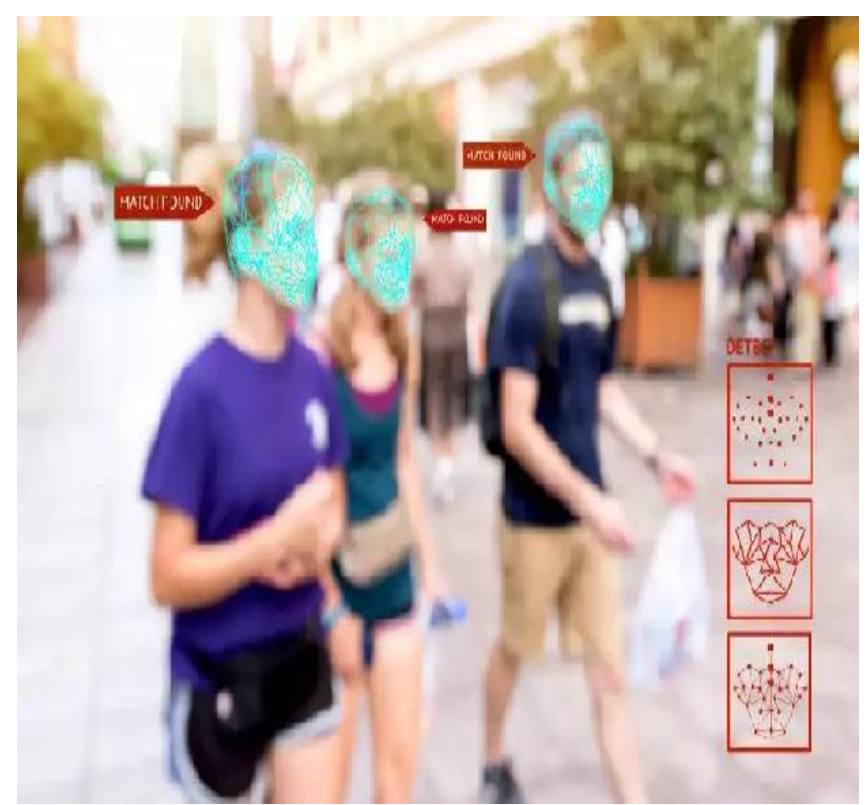

Fig-3: face recognised by TVs or LEDs or by mobile cameras when there walking in the public.

"The data record should be secured "such a way that it should never be misused by second hands, so it can get the greater effect of it.The Investigation unit will make sure that the data will phenomenally be safe and those criminals will not be benefited through these intelligent way of catching them. AI has the capacity to standardise the products feature like authentication, since in the verification of face stage it lets the investigation department to cross verify that the persons captured in the camera is matching with the image that was filed by the department has the same identity.
This isn't some far off technology tobe expected. It could be seen everywhere in country.

Apple is presenting facial ID abilities with itsiphone X. Apple utilizes facial information to open your telephone. In future it isn't utilized for it, could even help for perceiving facial data show in the cloud for military purposes. What's more, Apple isn't just the organization playing in our selfie-fixated society hoping to use our facial information. Different brands too are in the race.

Face recognition will one day replaces our password logins on our favourite social media apps - imagine logging in to facebook, instagram with your face, for example. when we have our phone in our hand a intelligence ofcreating consumer-focused face recognition technology for opening the closed app.

\section{CONCLUSION}

This survey has the information about face recognition.Its not that difficult task that it can't be implemented but still today the idea remain unsolved. Inspite of the new features being developed through artificial intelligence, having improvised algorithms still the ideaof facial recognition is still not been implemented everywhere. For example malls, restaurants, parking area , etc.Many algorithms are being built around this idea. As many strong feature extraction techniques have been developed, the challenge is to combine them so that to have a combined effect advantage. The novel methods used to gather visual information by providing simple images.Examples of this approach is genetic algorithms and artificial neural networks .Even the Boosting techniques. Diverse boosting techniques are being used and successfully applied to face recognition. One example is AdaBoost [15] in the well-known detection method developed by Viola and Jones. However in future the AI facial recognition will be implemented in large scale applications right from the mobiles in hand to the cctv which is hung in the public places. In future, the facial recognition controls all the major activities around the world, it would go to such an extent that even the information would be linked with thestudentID, digital driving license, passportor even national ID.Further the facial recordson the cloud using which one can get the information of a stranger or a criminal. Face recognition is also resulting in the other features, like expression recognition and body motion recognition.

Overall, face recognition techniques should be used in developed countries,so that by seeing it would grow in the technological aspects by this methods.

\section{REFERENCES}

[1]. Face Recognition Methods \& Applications. Divyarajsinh N. Parmar1 ,Brijesh B. Mehta2 1 P.G. Student of Computer Engineering 2Asst.Prof. Dept.of Computer Engineering C.U. Shah College of Engg. \& Tech Wadhwan city, India. 
[2]. VrushaliUbarhande, Alina-MadalinaPopescu, Horacio González-Vélez. Novel Data-Distribution Technique for Hadoop in Heterogeneous Cloud EnvironmentsCloud Competency Centre, Nat. Coll. of Ireland, Dublin, Ireland.

[3]. M. Turk. A random walk through eigenspace. IEICE Transactions on Information and Systems, E84-D(12):1586-1595, December 2001

[4]. K. Massy. "toyota develops eyelid-monitoring system". Cnet reviews, January 222008.

[5]. M. McWhertor. "sony spills more ps3 motion controller details to devs". Kotaku. Gawker Media., June 192009.

[6]. B. Dudley. "e3: New info on microsoft's natal - how it works, multiplayer and pc versions". The Seattle Times, June 3 2009.Technique for Hadoop in Heterogeneous Cloud Environments Cloud Competency Centre, Nat. Coll. of Ireland, Dublin, Ireland.

[7]. BIOID. Bioid face database. http://www.bioid.com/support/downloads/software/bioid-fac e-database.html. 